\title{
Möglichkeiten und Perspektiven häuslichen Trainings bei Patienten mit chronisch-obstruktiven Atemwegserkrankungen
}

\author{
A. R. Wewel \\ R. A. Jörres \\ D. Kirsten
}

\author{
Possibilities and Perspectives of Home-based Exercise Training in Patients with \\ Chronic Obstructive Pulmonary Diseases
}

\section{Zusammenfassung}

In Deutschland haben Rehabilitations- und Trainingsprogramme zur klinischen und funktionellen Verbesserung von Patienten mit chronisch-obstruktiven Atemwegserkrankungen eine lange Tradition. Dies gilt insbesondere für stationäre Programme. Aufbauend auf Erfahrungen des Auslandes wird jedoch inzwischen ambulanten oder häuslichen Programmen zunehmend Beachtung geschenkt. Ein zentraler Bestandteil der Programme ist körperliches Training. Dahinter steht die Erwartung, mit relativ geringem Aufwand den klinischen Zustand, sowie Lebensqualität, Krankheitsverlauf und Prognose des Patienten verbessern zu können. Erste Erfahrungen liegen - neben dem Asthma bronchiale - insbesondere bei der COPD vor. Die Daten legen nahe, dass körperliches Training die Exazerbationsraten senken kann und dieser Effekt mit minimalem Betreuungsaufwand erreichbar ist. Vordringliches Ziel künftiger Untersuchungen scheint daher die Entwicklung und Evaluierung praxisnaher, minimal aufwendiger Trainingsprogramme. Inwieweit diese Programme auf andere Lungenerkrankungen übertragbar sind, sollte ebenfalls geprüft werden.

\section{Abstract}

In Germany there is a long tradition of rehabilitation and exercise training programs to improve the clinical state and physical function of patients with chronic obstructive pulmonary diseases. This is particularly true regarding inpatient rehabilitation. Based on experiences gained in other countries, outpatient and home-based programs are currently getting more attention. One of the key components of pulmonary rehabilitation is exercise training which can be expected to exert direct and indirect beneficial effects on physical performance, health-related quality of life and even the progress of the disease. Most of the available experience refers to patients with COPD - besides those with bronchial asthma. The data available so far suggest that exercise training programs can reduce exacerbation rates and that this effect is achievable by minimal personal assistance. Thus the most important aim of future studies seems to be the development and evaluation of training programs which are efficient but also easy to implement and minimally expensive. Furthermore it needs to be studied whether such programs can be transferred to pulmonary disorders other than obstructive airway diseases.

\section{Einleitung}

Chronische Atemwegserkrankungen stellen lang verlaufende Krankheitsprozesse dar, die in der Regel eine lebenslange Therapie erfordern. Die fachgerechte Versorgung der Patienten wird - neben einer qualifizierten medikamentösen Therapie - durch eine darüber hinausgehende langfristige Unterstützung und Betreuung verbessert, die speziell auf die Charakteristika der jeweiligen Atemwegserkrankung abgestimmt ist. In den letzten Jahren wurden neben den klassischen Verfahren der stationären Reha-

Institutsangaben

Krankenhaus Großhansdorf, Zentrum für Pneumologie und Thoraxchirurgie, Großhansdorf

Korrespondenzadresse

Ärztin Alexandra R. Wewel · Krankenhaus Großhansdorf · Zentrum für Pneumologie und Thoraxchirurgie · Wöhrendamm 80 ·22927 Großhansdorf ·E-mail: a.wewel@pulmoresearch.de

Eingang: 13. April 2004 · Akzeptiert nach Revision: 10. Januar 2005

Bibliografie

Pneumologie 2005; 59: 328-336 c Georg Thieme Verlag KG Stuttgart · New York DOI $10.1055 / \mathrm{s}-2004-830196$

ISSN 0934-8387 
bilitation neue Ansätze vorgeschlagen oder erprobt und deren Indikation auf eine breitere Basis als bislang gestellt. Die vorliegende Übersicht soll einige der neueren Entwicklungen bzw. Überlegungen darstellen.

\section{Rehabilitation als unterstützende Maßnahme}

Die medizinische Rehabilitation stellt bei Patienten mit pneumologischen Erkrankungen die am weitesten verbreitete Form dar, um körperliche Leistungsfähigkeit und Lebensqualität nachhaltig zu verbessern [1 -4]. Darüber hinaus haben Rehabilitationsmaßnahmen auch das Ziel, die Patienten zu mehr Eigenaktivität anzuregen und sie auf Einstellungs- wie auch Verhaltensebene zum „Manager der eigenen Erkrankung“ zu schulen [5 - 7]. Die vorliegenden Überlegungen bzw. Denkmodelle zeigen, dass Einstellungs- und Verhaltensebene dabei verschiedene Aspekte darstellen [8-11]; insbesondere lernpsychologische Erkenntnisse sprechen für das Konzept, auf der Ebene der Einstellung die Motivation positiv zu beeinflussen und auf dieser Basis die Verhaltensänderung zu bewirken („Rubikon-Modell“) [12], die vermutlich ihrerseits die Motivation verstärkt $[11,13,14]$. Die somit geforderte Notwendigkeit aktiven Handelns fördert den individuellen Bezug zum Alltag des Patienten und trägt wesentlich dazu bei, den Nutzen rehabilitativer Maßnahmen in einen Gewinn an Lebensqualität zu überführen $[15,16]$.

Nachdem medizinische Rehabilitation bis vor wenigen Jahren praktisch ausschließlich stationär durchgeführt wurde, änderte sich dies nach der Forderung des Gesetzgebers, rehabilitative Maßnahmen „ambulant vor stationär“ durchzuführen. Mitte der 90er-Jahre begann so der Aufbau ambulanter rehabilitativer Versorgungseinrichtungen [17], die inzwischen parallel zur stationären Rehabilitation tätig sind. Neben einer erwarteten Reduktion der Gesamtkosten einer Erkrankung $[18,19]$ bieten ambulante Formen der Rehabilitation konzeptionell den Vorteil, dass bei wohnortnaher Durchführung der Patient im häuslichen - und oft auch parallel im beruflichen Umfeld - tätig bleibt. Auf diese Weise erreicht die ambulante Rehabilitation leichter das Ziel, den Bezug zum Alltag des Patienten herzustellen [20,21]; vor allem können mögliche Umsetzungsschwierigkeiten direkt bearbeitet werden. Einschränkend muss betont werden, dass die konzeptionellen Vorzüge der ambulanten Rehabilitation bislang nicht immer ausgeschöpft werden. Für einige Indikationen liegen mittlerweile Evaluationsstudien vor, beispielweise zum Vergleich ambulanter und stationärer Maßnahmen im Indikationsbereich Orthopädie und Kardiologie [18,22,23]. Hierbei zeigten sich keine wesentlichen Unterschiede der Wirksamkeit zwischen beiden Maßnahmen, sofern der ganzheitliche Rehabilitationsansatz auch im ambulanten Bereich berücksichtigt wurde. Bei schwerstgradigen pneumologischen Erkrankungen und/oder problematischer Persönlichkeitsstruktur ist jedoch aufgrund klinischer Erfahrungen der stationäre Ansatz vermutlich vorzuziehen.

Aufbauend auf Erfahrungen anderer Länder ist seit einigen Jahren der über das ambulante Training hinausführende Ansatz in der Diskussion, die Rehabilitation weitestgehend mittels häuslichen Trainings vorzunehmen. Dieses wird stationär oder ambulant eingeleitet und häuslich fortgesetzt oder erfolgt - nach neu- esten Vorstellungen - ausschließlich häuslich $[21,24,25]$. Zu bedenken ist allerdings, dass ein stationäres oder ambulantes Training besser definierbar ist als ein häusliches Training und hierin vermutlich eine Begrenzung dieses Ansatzes liegt. Ferner bedürfen zumindest einige Patienten möglicherweise des „Anschubs“ durch eine stationäre oder ambulante Maßnahme. In der Pneumologie sollte der Effizienzbeweis und Vergleich unterschiedlicher rehabilitativer Versorgungsformen zweckmäßigerweise im Rahmen der Qualitätssicherung erbracht werden [26-28].

\section{Trainingstherapie}

Unabhängig von der Art der Versorgungsform stellt körperliches Training im Rahmen der Therapie bei Patienten mit pneumologischen Erkrankungen eine wesentliche Grundlage der Rehabilitationsmaßnahme dar $[1,29,30]$. Allgemein kommen als Trainingsmöglichkeiten Kraft-, Koordinations- und Ausdauertraining zum Tragen [20,31,32]. Als Maximalanforderung können beispielsweise die Empfehlungen des American College of Sports Medicine (ACSM) für gesunde Menschen gelten; diese empfehlen die Durchführung eines kombinierten Trainings bei $60-90 \%$ der maximalen Herzfrequenz bzw. 50-80\% der maximalen Sauerstoffaufnahme über 20-45 Minuten 3-5mal pro Woche [33]. Für pneumologische Patienten beinhalten Trainingsprogramme ähnliche Vorstellungen; z.B. werden Fahrrad-, Geh- oder Laufbandtraining meist mit $60-75 \%$ der maximalen Leistungskraft des Patienten mehrfach wöchentlich bis zu mehrfach täglich durchgeführt [31,32]. Zusätzlicher Orientierungspunkt ist die Dyspnoe [34], beispielsweise das Erreichen von 75\% der maximalen Luftnot gemessen mittels Borg-Skala [35,36]. In einem anderen, eher auf Kraft ausgerichteten Trainingsmodell arbeitet der Patient an Trainingsgeräten mit ebenso gesteuerter Überprüfung der Leistungskraft. Vorschläge zur Wahl der Trainingsmodalitäten sowie zur Bemessung der Trainingsleistung bei Patienten mit verschiedenen Grunderkrankungen liegen seitens einiger Fachgesellschaften vor [37-39]. Die gegenwärtige Arbeit dient jedoch nicht der detaillierten Darstellung dieser Modalitäten, sondern versucht, häusliche und ambulante Trainingsprogramme als praktikable Optionen für die Zukunft einzuordnen.

Besonders erwähnenswert ist dabei, dass eine primär physiologisch orientierte Therapie nicht alleine von der Optimierung der physiologischen Effizienz profitiert, sondern auch von der Kombination der medizinischen mit einer verhaltenstherapeutischen Intervention. Körperliches Training hat zum einen direkte physiologische Wirkungen, insbesondere eine Leistungssteigerung und entweder direkt oder indirekt eine Reduktion der Atemarbeit $[29,32,40]$. Zum anderen erfahren die Patienten eine verhaltenstherapeutische Hilfestellung dergestalt, dass sie zentrale Beschwerden wie z.B. Atemnot besser einschätzen und damit ihre Leistungsfähigkeit stärker ausschöpfen können [20,41]. Ein verstärktes Selbstvertrauen, eine verbesserte Compliance sowie erweiterte Ressourcen zur Krankheitsbewältigung im Allgemeinen (Coping) sind in vielen Fällen die unmittelbare Folge $[42,43]$. Letztere scheinen wesentliche Faktoren bei der Reduktion gravierender Exazerbationen zu sein [44]. 
Training bei chronisch-obstruktiven

Atemwegserkrankungen

Die nationalen und internationalen wissenschaftlichen Veröffentlichungen der letzten Jahre belegen übereinstimmend die positiven Effekte eines körperlichen Trainings insbesondere bei chronisch-obstruktiven Atemwegserkrankungen [20,29-31,45-50]. Die oben beschriebenen Trainingsmodule wurden hierbei sowohl stationär als auch ambulant durchgeführt.

Im Bereich des häuslichen körperlichen Trainings werden zwar Elemente der Trainingsprogramme bzw. -module übernommen, jedoch ist es sinnvoll, in diesem Zusammenhang unter Training insgesamt alle Aktivitäten zu verstehen, die über die vom Patienten gewohnten körperlichen Aktivitäten hinausgehen. Es sind also auch Aktivitäten, die in das Alltagsleben integriert sind (täglicher Spaziergang, Gang zum Bäcker, Kiosk etc.), als Training zu werten und nicht nur solche, die bestimmte, vom Alltagskontext abgelöste Übungen umfassen, wie z.B. Laufbandtraining oder Training an Geräten. Damit ist auch ein Konzept des häuslichen Trainings denkbar, bei dem der Patient ausschließlich alltägliche Tätigkeiten vollbringt.

Entsprechend dem Schweregrad der Erkrankung sollten die für den individuellen Patienten in Betracht gezogenen Trainingskonzepte, einschließlich Kraft-, Koordinations- und Ausdauertraining, angepasst sein $[20,21,31]$. Bei der Auswahl der Modalitäten sollte man bedenken, dass die mögliche größere Effizienz bestimmter Konzepte bzw. Übungen im Vergleich zu anderen Konzepten in der Regel für Gruppen von Patienten gezeigt wurde, angesichts der Variabilität jedoch keineswegs für jeden einzelnen Patienten voraussagbar ist; stattdessen ist individuelle Anpassung gefordert. Erfolge des Trainings sind für alle Schweregrade der Erkrankung belegt, von leichter Obstruktion bis hin zu schwerer respiratorischer Insuffizienz [20]. In jedem Fall führt Training zu einer Verbesserung der Lebensqualität sowie zu einer gesteigerten Kurz- wie Ausdauerleistung.

Unter den bisherigen Arbeiten zur Wirkung eines körperlichen Trainings in der Pneumologie sind diejenigen zur COPD von besonderem Interesse. Dies gilt nicht zuletzt angesichts der im Vergleich zum Asthma in der Regel stärker begrenzten Therapieoptionen. In der vorliegenden Übersicht soll daher das Asthma weitgehend ausgeklammert sein, zumal Erfahrungen zum Training bei Patienten mit Asthma, insbesondere bei Kindern, bereits ausführlich und zusammenfassend berichtet wurden $[51,52]$.

Der akute positive Effekt kurzzeitiger Trainingsprogramme bei Patienten mit COPD ist vielfach belegt. In eigenen Arbeiten konnten wir zeigen, dass ein nur 10-tägiges, stationär durchgeführtes Trainingsprogramm bei Patienten mit mittel- bis schwergradiger COPD nach einer Exazerbation ihrer Erkrankung eine markante Verbesserung der körperlichen Leistungsfähigkeit hervorrief, ohne mit wesentlichen Veränderungen der Lungenfunktion einherzugehen [53]. Ähnliche, wenngleich im Ausmaß geringere Effekte eines 10-tägigen stationären Trainingsprogramms fanden sich bei Patienten mit COPD in stabilem Zustand [54]. Möglicherweise erklärt sich der größere Benefit der erstgenannten Patienten durch die Erfahrung ihrer vorangegangenen Exazerbation, indem sie besonders motiviert waren, das Trainingsprogramm nach ihrer klinischen Stabilisierung zu beginnen. Nur mittels der Annahme einer gesteigerten Motivation sowie einer verbesserten, d.h. ökonomischeren muskulären Koordination erscheint beispielsweise ein Gewinn an Gehstrecke von nahezu 100\% innerhalb von nur 10 Tagen erklärbar.

Nach allen eigenen Erfahrungen ist in der Tat nur ein Teil der Trainingseffekte auf eine direkte Erhöhung der Leistungsfähigkeit zurückzuführen. Ein wesentlicher Teil beruht auf der Tatsache, dass die Patienten ihre Leistungsfähigkeit besser einschätzen und einsetzen können und daher beispielsweise am Ende der Trainingsperiode sogar eine erhöhte Dyspnoe in Kauf nehmen. Vor dieser Leistung wären sie ohne die Erfahrung des Trainings zurückgeschreckt, wie die Daten der Kontrollgruppe belegten $[49,55]$.

Die Befunde zum Training sind deshalb von besonderem Interesse, weil die Prognose des Krankheitsverlaufs bei Patienten mit schwergradiger COPD wesentlich von der körperlichen Leistungsfähigkeit abhängt $[50,56]$. Dementsprechend sind langfristig angelegte Programme notwendig, die gewährleisten, dass ein initial erzielter Gewinn über einen längeren Zeitraum beibehalten oder sogar ausgebaut werden kann. In der Tat konnten die positiven Effekte eines Kurzzeittrainings durch eine weitmaschige häusliche Betreuung nach Entlassung aus dem Krankenhaus gehalten werden [57], und nach einem Zeitraum von anderthalb Jahren ließ sich sogar eine Verminderung der Zahl krankheitsassoziierter Exazerbationen nachweisen [58]. Man kann davon ausgehen, dass in den genannten Studien die Akzeptanz des häuslichen Trainings durch die Tatsache, dass dieses als Teil täglicher Lebensaktivitäten konzipiert war, sowie durch das mit der wiederholten Kontrolle des Trainings und klinischen Befindens verbundene Gefühl der Zuwendung gesteigert wurde. In einer groß angelegten Studie wurde in Übereinstimmung damit gefunden, dass eine geringe körperliche Aktivität im Alltag einen wesentlichen Risikofaktor für das Auftreten einer Exazerbation darstellte [59].

Viele der untersuchten langfristigen Trainingsprogramme erforderten allerdings entweder lange stationäre Aufenthalte oder bei ambulanter Rehabilitation viele Visiten in relativ kurzen Abständen [21]. Bei Beachtung der Kostenlage im Gesundheitssystem ist es daher ein sinnvolles Ziel künftiger Studien, minimal aufwendige längerfristige Programme zu entwickeln und diese sowohl auf ihre Effizienz als auch auf ihre Kosten hin zu evaluieren.

\section{Vergleich von Betreuungsmodalitäten}

Inzwischen liegt eine Reihe von Daten vor, in denen verschiedene Grade der Betreuung von häuslichen Trainings bei Patienten mit COPD miteinander verglichen wurden $[60,61]$. In einer eigenen Untersuchung ergab der Vergleich verschiedener Betreuungsmodalitäten, dass zur Beibehaltung der positiven Effekte auf Leistungsfähigkeit und Lebensqualität unter häuslichen Bedingungen eine postalische Betreuung ausreichend war [54]. Hausbesuche oder der Versuch einer Kontrolle des häuslichen Trainings durch Pedometer erbrachten keine weitere Verbesserung. Die Integration der dargestellten Trainingskonzepte mit 
denjenigen der Lungensportgruppen ist zu befürworten [62,63], zumal in diesen Gruppen von einer hohen Motivation auszugehen ist. Allerdings werden im Einzelfall die örtlichen Gegebenheiten, die beispielsweise durch Verkehrsanbindungen und Siedlungsdichte (Stadt vs. Land) gegeben sind, maßgeblich für die kombinierte oder sequenzielle Durchführung sein.

Zur Zeit ist die Zahl der Institutionen in Deutschland, die ein regelmäßiges häuslich betreutes Trainingsprogramm für Lungenund Atemwegserkrankte anbieten, nicht flächendeckend. Bei der Beurteilung derartiger Programme ist zu beachten, dass eine optimale Versorgung der Erkrankten erst gegeben ist, wenn rehabilitative Maßnahmen wie Krankheitsschulung, Raucherentwöhnung und Atemtherapie neben körperlichem Training und medikamentöser Therapie berücksichtigt werden [20,62]. Die Umsetzung eines derartigen integrativen Konzepts in die Praxis steht in Deutschland erst am Anfang. Solange diese Konzepte noch nicht hinreichend mit Daten belegt sind und der wissenschaftlichen Evaluierung bedürfen, werden vermutlich auch Programme, die auf ein alleiniges häusliches kontrolliertes Training abzielen, nicht ganz ohne einen ambulanten Part auskommen, der der Charakterisierung der Patienten, dem Start des Programms sowie einer weitmaschigen Überwachung durch Messungen im Labor dient.

Die für Diabetes mellitus und Mammakarzinom bereits eingeführten DMP-Programme (Disease Management) beinhalten bereits - unter allen Vorbehalten, die u.a. in der noch mangelnden Erfahrung begründet sind - eine systematische, multiprofessionelle und patientenzentrierte Versorgung, die vorwiegend auf ambulanter Versorgung aufbaut und die Integration in den Alltag herbeiführen soll $[64,65]$. Die USA nimmt in dieser Hinsicht eine Vorreiter-Rolle ein [66,67]. Dabei kommt dem Pflegepersonal eine tragende Rolle zu [20,67]. Es steht zu erwarten, dass ambulante Betreuung und Rehabilitation auch in Deutschland stärker als bisher durch nichtärztliches Personal geleistet werden.

\section{Häusliches Training}

Im Vergleich zu konventionellen Rehabilitationsmaßnahmen wird nach unserer Auffassung bisher die Möglichkeit zu wenig genutzt, ein Training weitestgehend zu Hause durchzuführen, ohne vorher den Prozess einer umfänglichen Rehabilitationsmaßnahme zu durchlaufen. Zwar bestehen - im Gegensatz zu den ambulanten Ansätzen - zur Zeit die erforderlichen organisatorischen Strukturen noch nicht in zureichendem Maße, jedoch wird man möglicherweise auf die Erfahrungen einiger der vorliegenden wissenschaftlichen Arbeiten zurückgreifen können, soweit diese bereits im Hinblick auf eine spätere Realisierbarkeit konzipiert waren $[54,58]$.

\section{Überwachung häuslichen Trainings}

Moderne Betreuungs- bzw. Nachsorgeprogramm bedürfen heute der Computerunterstützung zur Diagnostik, Interventionssteuerung, Dokumentation und Evaluation. Ziel ist es, die Daten eines Patienten in einer möglichst leicht verfügbaren und auswertbaren Form zur Verfügung zu haben und mit möglichst geringem
Aufwand eine Effizienzkontrolle durchzuführen [68,69]. In Verbindung damit ist die Versorgungskontinuität am besten gewährleistet durch die Entwicklung spezieller, auf diese Belange abgestimmten Pflegesysteme (sog. „Sekundärprävention“), die zum Ziel haben, durch begrenzte, ökonomisch vertretbare Interventionen das Auftreten gravierender Erkrankungsfolgen zu minimieren $[70,71]$.

Ein Trainingsprogramm kann im einfachsten Fall telefonisch mittels regelmäßiger Anrufe oder schriftlich durch regelmäßige Ausgabe und Einsendung von Tagebüchern überwacht werden. Ferner können Hausbesuche hilfreich sein. Sie scheinen nach unseren eigenen, an einer kleinen Gruppe von Patienten mit COPD gewonnenen Daten [54] keine wesentliche Verbesserung der Trainingserfolge - beispielsweise durch eine Steigerung der Motivation - zu bewirken. Initiale Hausbesuche können dennoch die Durchführung des Programms erleichtern, da eine geschulte Kraft die individuellen Gegebenheiten des Patienten in Augenschein nehmen und demgemäß Ratschläge zur Durchführung des Trainings erteilen kann.

Möglichkeiten zur Steuerung des Trainings sind gegeben durch die Borg-Skala als Maß der Dyspnoe, sowie physiologische Messgrößen wie Herzfrequenz, Sauerstoffaufnahme und Laktatkonzentration [31,72]. Allerdings hat bei schwer- und schwerstgradig erkrankten Patienten nach unserer Auffassung die Trainingssteuerung hauptsächlich die Aufgabe, das Training beizubehalten, und dient erst in zweiter Linie der Anpassung an augenblickliche Gegebenheiten. Daten aus der häuslichen Betreuung von Patienten mit COPD zeigten keine Beziehung zwischen dem Ausmaß der täglichen Aktivität und der Leistungsfähigkeit bei Visiten im Krankenhaus [57]. Entscheidend war nur, dass überhaupt ein Training stattfand, wie der Vergleich mit der Kontrollgruppe ergab. Es sollte nach Möglichkeit zu den Zielen der Schulung des Patienten gehören, die augenblickliche Anpassung weitgehend selbst leisten zu können. Eigene Erfahrungen sprechen dafür, dass dem Patienten ein hohes Maß an Eigenständigkeit übertragen werden kann und gleichzeitig positive Effekte selbst über einen längeren Zeitraum zu erzielen sind $[57,73]$.

Die Betreuung eines Trainings „aus der Ferne“ bietet sich an, ggf. mit telemedizinischen Konzepten verknüpft zu werden und auf diese Weise die Dokumentation des Trainings zu erleichtern $[68,69]$. Eine derartige tägliche Auswertung und Datenübermittlung, ggf. unter Mithilfe von Pedometern oder Aktographen, ist bislang nicht untersucht. Da Lungenfunktion und Krankheitszustand bei der COPD nicht so eng gekoppelt scheinen wie beim Asthma, scheint die Hypothese nicht abwegig, dass beginnende Exazerbationen bzw. Verschlechterungen des Allgemeinzustandes bei Patienten mit COPD sich in einer auffälligen Reduktion der Aktivität niederschlagen könnten, selbst dann, wenn sie in der Lungenfunktion noch nicht eindeutig zu erkennen sind. Praktische Erfahrungen hierzu konnten in Untersuchungen mit kardiologischen Patienten gesammelt werden, wobei sich Verbesserungen in der Patientenversorgung zeigten [74-76]. Daher könnte ein telemedizinischer Betreuungsmodus eventuell auch Vorteile in der Langzeitbetreuung bei Patienten mit Atemwegserkrankungen mit sich bringen. 
Telefonische Programme spielen insofern eine wesentliche Rolle in der Nachsorge, als sie den Vorteil haben, relativ kostengünstig zu sein $[77,78]$. Fahrtkosten und -zeiten entfallen, und dennoch ist ein persönlicherer Kontakt als bei postalischer oder telemedizinischer Betreuung möglich. Entsprechende Erfahrungen werden in Deutschland zur Zeit beispielsweise im Rahmen einer multizentrischen, prospektiven, kontrollierten Studie an 200 Koronarpatienten gesammelt, die nach Entlassung aus der stationären AHB von in der kardiologischen Rehabilitation erfahrenen Studienschwestern $1 \mathrm{Jahr}$ lang telefonisch nachbetreut und mit 200 Patienten einer Kontrolle verglichen werden [79]. Endpunkte der Beobachtung sind in dieser Studie koronare Risikofaktoren wie Gesamtcholesterin, systol. Blutdruck, HbA1c, Rauchen sowie eine leitliniengerechte Medikation. Die erforderlichen strukturellen Rahmenbedingungen wurden in der Planungsphase geschaffen, indem technisch-organisatorisch, pflegewissenschaftlich, inhaltlich und juristisch die relevanten Aspekte vorab festgelegt wurden. Diese Studie könnte, neben anderen, als konkreter Ausgangspunkt für die Realisierung analoger Projekte bei Patienten mit chronischen Atemwegserkrankungen wie COPD dienen.

Entsprechende Programme für Patienten mit Diabetes, Adipositas oder Osteoporose sind in der Erprobung. Teilweise werden diese durch die gesetzlichen oder privaten Krankenversicherungen durchgeführt, teils werden externe Dienstleistungsfirmen, meist medizinische Call-Center, u.a. vom Bundesministerium für Gesundheit im Rahmen von Studien damit beauftragt $[80,81]$.

\section{Anforderungen bei Betreuung per Telefon}

Hinsichtlich der telefonischen Betreuung spielt neben der Vertrauenswürdigkeit des jeweiligen Anrufers die Qualität des Telefonates eine entscheidende Rolle für die Akzeptanz seitens des Patienten und hat direkte Auswirkungen auf den gesundheitspräventiven langfristigen Erfolg [77,78]. Das Telefonpersonal muss darin geübt werden, anhand telefonischer Kontakte Schwierigkeiten und Krisen des Patienten, z. B. akute physiologische Komplikationen wie Herzinsuffizienz und respiratorische Insuffizienz, aber auch emotional bedeutsame Faktoren, wie eine (larvierte) akute Depression, zu erkennen und adäquat zu reagieren. Ob dies zur Zeit allein durch formale Screeningverfahren geleistet werden kann, ist angesichts des erforderlichen Evaluierungsaufwandes fraglich. Das Personal muss sicherlich die passenden Verhaltens- und Vorgehensweisen sowohl durch besondere Schulung als auch aus allgemeiner Lebenserfahrung (Einschätzung anderer Personen) erlernen. Vorteilhaft scheint hier der Einsatz von Pflegepersonal mit seiner speziellen pflegerischen Erfahrung zu sein.

\section{Alktivität als Maß des Trainingseffelkts}

Die körperliche Aktivität der Patienten, als zentrale Kenngröße der Aktionsfähigkeit und -bereitschaft im Alltag, ist bislang in der Regel nur summarisch über Fragebogendaten erfasst worden. In einer Reihe von Untersuchungen wurden auch die Gesamtaktivität zählende Pedometer verwandt [82-84]. Es steht zu vermuten, dass ein Profil der Aktivität über den Tag in besonders enger Beziehung zur Lebensqualität steht, da es nicht nur die mittlere Leistung, sondern auch die maximal erbrachten Leistungen und ihre Häufigkeit dokumentiert. Derartige Daten liegen bisher praktisch nicht vor. Ferner wäre es von Interesse, zu prüfen, inwieweit Interventionen, die sowohl eine physiologische als auch eine psychologische Komponente beinhalten, insbesondere telefonisch betreute häusliche Trainingsprogramme, Wirkungen auf das Aktivitätsprofil haben. Ferner stellt sich die Frage, inwieweit diese Interventionen einen positiven Effekt auf die Lebensqualität haben. Auch wenn die Korrelationen zwischen objektiven und subjektiven Größen im Allgemeinen nur gering und eingeschränkt praktisch verwertbar sind, würde die Erfolgskontrolle vermutlich durch die Analyse des Aktivitätsprofils gewinnen. Es muss allerdings berücksichtigt werden, dass die gemessene Aktivität nur einen Teilaspekt des klinischen Gesamtzustandes des Patienten darstellt und daher, ähnlich den physiologischen Messgrößen, zum Zwecke der Bewertung und Prädiktion nur im Kontext anderer Variablen gesehen werden darf.

\section{Verhalten des Patienten}

Über eine Verbesserung der physiologischen Größen hinaus ist die Beeinflussung des Verhaltens des Patienten zentrales Ziel der Betreuung [41]. Bei Patienten mit COPD steht naturgemäß die Raucherentwöhnung an erster Stelle [82]. Diese hat vermutlich allein über das gesteigerte Gesundheitsbewusstsein bereits einen günstigen Einfluss auf andere verhaltensabhängige Risikofaktoren, wie ungesunde Ernährung und Mangel an Bewegung [82]. Wichtig erscheint, sich vor Augen zu führen, dass das Verhalten des chronisch kranken Patienten durch viele Faktoren bestimmt ist, nicht nur - oder sogar am wenigsten - physiologische Faktoren. Dies betrifft insbesondere den Patienten mit COPD, dem es durch den langsamen Krankheitsverlauf offenbar über einen langen Zeitraum gelingt, die fortlaufende Einschränkung seiner Leistungsfähigkeit in seinen Lebensablauf zu integrieren oder diese als altersbedingten Verlauf zu kaschieren. Somit wäre es nicht gerechtfertigt zu erwarten, dass beispielsweise eine Verbesserung der Lungenfunktion sich unmittelbar in eine solche des Aktionsradius umsetzt, wenn nicht gleichzeitig motivationssteigernde Faktoren wirksam sind. Daten unseres Labors zeigen, dass zumindest ein stationäres Kurzzeittraining sich in einer Steigerung der körperlichen Aktivität auch außerhalb der Trainingszeiten niederschlägt, jedoch scheint auch dieser Effekt primär über eine gesteigerte Motivation vermittelt [73]. Inwieweit er nach Beendigung eines Programms anhält, ist zur Zeit nicht bekannt.

\section{Zielgrößen der Effektivität eines Trainings}

Zwar bestehen positive Korrelationen zwischen Dyspnoeempfinden, Lebensqualität und psychologischen Kenngrößen einerseits und physiologischen bzw. leistungsbezogenen Messgrößen andererseits [20,31] doch sind die Korrelationskoeffizienten in vielen Fällen ungeachtet der statistischen Signifikanz so gering, dass im individuellen Fall keineswegs eine eindeutige Voraussage von einer Messgröße auf eine andere möglich ist. Dies legt die Annahme nahe, dass nicht eine einzelne Zielgröße zum Orientie- 
rungswert der ambulanten oder häuslichen Betreuung bzw. des Trainings erklärt werden sollte, sondern eine Verbesserung im Ganzen anzustreben ist. Möglicherweise werden bei genügend großen Fallzahlen und Messgrößen multivariate statistische Verfahren helfen können, einen Gesamtscore zu definieren, der bei der Mehrzahl der Patienten mit der subjektiven und/oder objektiven Besserung konform geht. Vermutlich müssen in einem derartigen Score auch die psychologischen Profile der individuellen Patienten Eingang finden. Es stellt sich die Frage, inwieweit das Wissen um diese sowohl krankheitsspezifischen als auch individuellen Profile für die Optimierung des Trainingserfolges genutzt werden kann. Diese Frage erscheint angesichts knapper Ressourcen im Gesundheitssystem von großem praktischem Interesse.

Die krankheitsbezogenen Exazerbationen stellen eine der zentralen Zielgrößen einer Intervention mittels eines ambulanten oder häuslichen Trainingsprogramms dar [62]. Dass die Exazerbationsrate bei Patienten mit schwergradiger COPD durch ein minimal aufwendiges häusliches Betreuungs- und Trainingsprogramm signifikant gesenkt werden kann, wurde an einer kleinen Gruppe von Patienten bereits gezeigt [58]. Analoge Daten liegen auch mit Programmen des Self-Management und telefonischer Nachbetreuung vor [85].

Eine weitere fundamentale Zielgröße ist durch die Lebensqualität gegeben [62]. Zwar finden sich Korrelationen zwischen Lebensqualität und physiologischen Größen, doch sind diese in der Regel so gering, dass die Lebensqualität als eigenständige Variable und als eigenständiger Wert angesehen werden muss. Die vorliegenden Studien [86 - 88] ergaben als konsistenten Befund, dass ein häusliches oder ambulantes Training die Lebensqualität bei Patienten mit COPD verbessert und dies die Mehrzahl der verschiedenen psychologischen Dimensionen betrifft, die bei der Auswertung herangezogen wurden.

\section{Mögliche Kostenredultion durch Training}

Nur wenn die Interventionen auf Dauer einen gesundheitlichen Benefit für die Teilnehmer erbringen, werden sie längerfristig gerechtfertigt und etabliert werden können. Selbstverständlich ist es auch das Ziel der Betreuungsprogramme, letztlich eine Kostenreduktion herbeizuführen [89]. Erreicht werden soll dies durch den Rückgang der Zahl stationärer Aufenthalte einerseits und die Vermeidung von Überversorgung andererseits $[58,90]$. Auch sollte sich die Aufenthaltsdauer eventuell erforderlicher stationärer Aufenthalte verringern lassen, wenn eine anschließende effiziente Weiterversorgung durch Nachsorgeprogramme gesichert ist [20,57]. Es steht zu erwarten, dass die Gesamtheit dieser Faktoren - neben einer möglichen, gleichzeitigen Verringerung des Medikamentenbedarfs - zu einer Reduktion der Kosten beiträgt.

\section{Notwendigkeit von Studien/Evaluierung}

Aus dem Gesagten geht hervor, dass es trotz vieler publizierter Studien zum stationären, ambulanten und häuslichen Training bzw. entsprechender Betreuung an Daten mangelt, die sich auf Programme beziehen, die unter den gegebenen Kostenrestriktio- nen verwirklichbar und zugleich effektiv sind. Dies betrifft auch die langfristige Kosten/Nutzen-Relation hinsichtlich des Effektes auf den Verlauf der Erkrankung sowie den Einsatz von kostengünstigeren Betreuungsmodalitäten unter möglichst geringem Personalaufwand. In dieser Hinsicht sind auch Forschungs- bzw. Evaluierungsprogramme wünschenswert, die von den zuständigen Kostenträgern des Gesundheitssystems selbst finanziert werden, da diese langfristig von den erwarteten Erfolgen profitieren sollen. Diese Entwicklung zeigt sich bereits am Engagement vor allem der privaten Krankenkassen bezüglich der Etablierung von Call-Centers $[80,81,91]$.

\section{Übertragbarkeit auf andlere Atemwegs- und} Lungenerkrankungen

Pulmonale Rehabilitationsprogramme wurden bisher fast ausschließlich bei Patienten mit chronisch-obstruktiven Lungenerkrankungen wie COPD oder Asthma durchgeführt. Patienten mit schwergradigen nicht-obstruktiven Lungenerkrankungen - wie neuromuskuläre Erkrankungen, Zustand nach größeren chirurgischen Eingriffen wie Pneumonektomie bzw. Transplantation oder restriktive Lungenerkrankungen - sind in ihrer Leistungsfähigkeit ähnlich beeinträchtigt wie Patienten mit COPD und weisen daher möglicherweise auch ein Potential für eine klinische und funktionelle Verbesserung auf. Abhängig vom Schweregrad der Erkrankung besteht auch bei diesen Patientengruppen Belastungsdyspnoe und in variablem Umfang eine körperlich-muskuläre Schwäche infolge krankheitsbedingter Inaktivität $[20,92]$. Vor diesem Hintergrund wird auch bei Patienten mit nicht-obstruktiven Lungenerkrankungen der Versuch der Funktionsund Lebensqualitätsverbesserung mittels rehabilitativer Maßnahmen empfohlen [93]; jedoch nehmen nur ca. 10\% der Patienten an Rehabilitationsprogrammen teil.

Unter den restriktiven Lungenerkrankungen umfasst die Gruppe der interstitiellen Lungenerkrankungen eine Reihe ätiologisch heterogener Erkrankungen [94,95]. Die Prävalenz dieser Erkrankungen ist deutlich niedriger als diejenige der obstruktiven Erkrankungen. Für die häufigste, die Sarkoidose, liegt die Prävalenz in Deutschland bei 50 auf 100000 , für die idiopathische Lungenfibrose wird sie auf ca. 6 auf 100000 geschätzt [95]. Beide Erkrankungen haben gemeinsame klinische und funktionelle Kennzeichen [96], inklusive einer reduzierten Belastbarkeit [97], für die eine arterielle Hypoxämie verantwortlich gemacht wird $[98,99]$. Somit bestehen gewisse funktionelle Parallelen zur COPD. In ähnlicher Weise ist der positive Effekt einer Sauerstoffgabe dokumentiert $[98,100]$. Allerdings gibt es keine Daten zur Effektivität eines körperlichen Trainingsprogrammes bei Patienten mit nicht-obstruktiven Lungenerkrankungen. Die American Thoracic Society beispielsweise empfiehlt die Anwendung multidisziplinärer Rehabilitationsprogramme auch bei diesen Patienten, ohne sich jedoch auf konkrete Daten zu beziehen [101].

Obgleich die Studien zu derartigen Rehabilitationsprogrammen, deren Daten zur Zeit vorliegen, in der Regel nur geringe Patientenzahlen und ein gemischtes Patientengut umfassten [2,3], konnten bereits positive Effekte in Form von funktionellen Verbesserungen aufgezeigt werden. Auch ist lange bekannt, dass präoperativ vor Lungentransplantationen Patienten mit idiopa- 
thischer Lungenfibrose von einem Training profitieren [92]. Somit könnte ein ambulantes bzw. häusliches Trainingsprogramm bei derartigen Patienten durchaus erfolgreich sein, auch wenn im Vergleich zur COPD durch den progressiven Verlauf der Erkrankung langfristige Effekte weniger wahrscheinlich sind. Zur Zeit untersuchen wir die Wirkungen eines derartigen Programms in einer prospektiven Studie über den Zeitraum von 18 Monaten bei einer großen Zahl von Patienten [102].

\section{Perspektiven für die Zukunft}

Ambulante und/oder häusliche Trainingsprogramme haben nachgewiesenermaßen positive Effekte insbesondere bei Patienten mit chronisch-obstruktiven Atemwegserkrankungen. Allerdings wurden in den entsprechenden Studien häufig Konzepte bzw. Programme eingesetzt, die einen relativ hohen Aufwand erforderten. Um derartige Programme als integralen Bestandteil des Gesundheitssystems zu implementieren, scheint es notwendig, ihrer Realisierbarkeit unter minimalen Kosten stärkere Beachtung zu schenken. Zumindest unter Kostengesichtspunkten erscheinen häusliche, fernbetreute Programme besonders erfolgversprechend. Unter Einsatz der modernen Datentechnik sollten eine Vernetzung der zuständigen Institutionen (Kliniken, ambulante Zentren, Lungensportgruppen) und eine laufende aussagekräftige Effizienzkontrolle möglich sein. Auch ist in Betracht zu ziehen, andere als nicht-obstruktive Lungenerkrankungen mit derartigen Programmen anzugehen.

\section{Literatur}

${ }^{1}$ Goldstein RS, Gort EH, Stubbing D et al. Randomised controlled trial of respiratory rehabilitation. Lancet 1994; 344: $1394-1397$

2 BTS Statement. Pulmonary Rehabilitation. Thorax 2001; 56: 827-834

${ }^{3}$ ATS. Pulmonary Rehabilitation - 1999. Am J Respir Crit Care Med 1999; 159: 1666- 1682

${ }^{4}$ Nield M. Pulmonary rehabilitation: the critical outcomes. J Rehabil Res Dev 2003; 40 (5 Suppl 2): vii - viii

${ }^{5}$ Lacasse Y, Guyatt GH, Goldstein RS. The components of a respiratory rehabilitation program: a systematic overview. Chest 1997; 111: $1077-1088$

${ }^{6}$ Worth H, Dhein Y. Does patient education modify behaviour in the management of COPD? Patient Education and Counseling 2004; 52: 267-270

${ }^{7}$ Emery CF, Schein RL, Hauck ER et al. Psychological and cognitive outcomes of a randomised trial of exercise among patients with chronic obstructive pulmonary disease. Health Psychol 1998; 17: 232-240

${ }^{8}$ Bandura A. Social learning theory. Englewood Cliffs (NJ): Prentice Hall, 1977

${ }^{9}$ Bandura A. Self-efficacy: toward a unifying theory of behavioral change. Psychol Rev 1977; 84: 191-215

${ }^{10}$ Bandura A. The self system in reciprocal determinism. Am Psychol 1978; 33: 344-358

${ }^{11}$ Bourbeau J, Nault D, Dang-Tan T. Self-management and behaviour modification in COPD. Patient Education and Counseling 2004; 52: $271-277$

${ }^{12}$ Heckhausen H, Gollwitzer PM. Thought contents and cognitive functioning in motivational vs. volitional states of mind. Motivation \& Emotion 1987; 11: $101-120$

${ }^{13}$ Atkins CJ, Kaplan RM, Timms RM et al. Behavioral exercise programs in the management of chronic obstructive pulmonary disease. J Consult Clin Psychol 1984; 52: 591 - 603

${ }^{14}$ Eiser N, West C, Evans S et al. Effects of psychotherapy in moderately severe COPD: a pilot study. Eur Respir J 1997; 10: 1581 - 1584
${ }^{15}$ Morgan MD, Singh SJ, Hyland ME. The relationship between physical activity and quality of life in chronic lung disease. Eur Respir Rev 1997; 7: $57-59$

${ }^{16}$ Clough P, Harnisch LA, Cebulski P et al. Method for individualizing patient care for chronic obstructive pulmonary disease patients. Health Soc Work 1987; 12: 127-133

${ }^{17}$ Koch U, Bürger W. Ambulante Rehabilitation - Ziele, Voraussetzungen und Angebotsstruktur. Schriftenreihe zum Gesundheits- und Sozialwesen. Bundesverband Deutscher Privat-Krankenanstalten 1996

18 Bürger W, Dietsche S, Morfeld M et al. Ambulante und stationäre orthopädische Rehabilitation - Ergebnisse einer Studie zum Vergleich der Behandlungsergebnisse und Kosten. Die Rehabilitation 2002; 41: $92-102$

${ }^{19}$ Güell R, Casan P, Belda J. Long-term effects of outpatient rehabilitation of COPD: A randomized trial. Chest 2000; 117: 976-983

20 Tiep BL. Disease management of COPD with pulmonary rehabilitation. Chest 1997; 112: $1630-1656$

${ }^{21}$ Strijbos JH, Postma DS, Altena R van et al. A comparison between an outpatient hospital-based pulmonary rehabilitation program and a home-care pulmonary rehabilitation program in patients with COPD. A follow-up of 18 months. Chest 1996; 109: 366 - 372

22 Wolf N, Törne I von, Weber-Falkensammer H. Evaluation der ambulanten orthopädisch-traumatologischen Rehabilitation der Ersatzkassen. Ein Vergleich mit der stationären Rehabilitation. Die Rehabilitation 1999; 38 (Suppl.1): 44-51

${ }^{23}$ Gysan DB, Wendt T. Ambulante/teilstationäre kardiologische Rehabilitation. Herz 1999; 24 (Suppl. 1): 44-56

${ }^{24}$ McGavin CR, Gupta SP, Lloyd EL et al. Physical rehabilitation for the chronic bronchitic: results of a controlled trial of exercises in the home. Thorax 1977; 32: 307-311

${ }^{25}$ Wijkstra PJ, Altena R van, Kraan J et al. Quality of life in patients with COPD improves after rehabilitation at home. Eur Respir J 1994; 7: $269-273$

${ }^{26}$ Bergmann K, Fischer J, Schmitz M et al. Die stationäre pneumologische Rehabilitation für Erwachsene. Zielsetzungen - diagnostische und therapeutische Standards - Forschungsbedarf. Statement der Sektion Prävention und Rehabilitation. Pneumologie 1997; 51: $523-532$

27 Petro W. Pneumologische Prävention und Rehabilitation. Ziele - Methoden - Ergebnisse. Berlin: Springer Verlag, 2000

${ }^{28}$ Sachverständigenrat für Konzertierte Aktion im Gesundheitswesen. Bedarfsgerechtigkeit und Wirtschaftlichkeit, Band III - Über-, Unterund Fehlversorgung, Gutachten. 2000/2001

${ }^{29}$ Griffiths TL, Burr ML, Campbell IA et al. Results at 1 year of outpatient multidisciplinary pulmonary rehabilitation: a randomised controlled trial. Lancet 2000; 355: 362-368

${ }^{30}$ Sassi-Dambron DE, Eakin EG, Ries AL et al. Treatment of dyspnea in COPD: a controlled clinical trial of dyspnea management strategies. Chest 1995; 107: 724-729

${ }^{31}$ Spruit MA, Troosters T, Trappenburg JCA et al. Exercise training during rehabilitation of patients with COPD: A current perspective. Patient Education and Counseling 2004; 52: $243-248$

${ }^{32}$ Gigliotti F, Romagnoli I, Scano G. Breathing retraining and exercise conditioning in patients with chronic obstructive pulmonary disease (COPD): a physiological approach. Respiratory Medicine 2003; 97: 197-204

${ }^{33}$ American College of Sports Medicine Position Stand. The recommended quantity and quality of exercise for developing and maintaining cardiorespiratory and muscular fitness, and flexibility in healthy adults. Med Sci Sports Exerc 1998; 30: 975 - 991

${ }^{34}$ Meek PM, Lareau SC. Critical outcomes in pulmonary rehabilitation: assessment and evaluation of dyspnea and fatigue. J Rehabil Res Dev 2003; 40 (5 Suppl 2): 13-24

${ }^{35}$ Mejia R, Ward J, Lentine $\mathrm{T}$ et al. Target dyspnea ratings predict expected oxygen consumption as well as target heart rate values. Am J Respir Crit Care Med 1999; 159: 1485-1489

${ }^{36}$ Horowitz MB, Littenberg B, Mahler DA. Dyspnea ratings for prescribing exercise intensity in patients with COPD. Chest 1996; 109: $1169-1175$

37 American Association of Cardiovascular and Pulmonary Rehabilitation. Guidelines for Pulmonary Rehabilitation programs, 2nd edn. Champain: Human Kinetics, 1998

${ }^{38}$ Deutsche Hochdruckliga. Empfehlungen zur Hochdruckbehandlung 2001. www.paritaet.org/hochdruckliga 
${ }^{39}$ Arbeitsgemeinschaft der Wissenschaftlichen Medizinischen Fachgesellschaften (AWMF). Prävention, Standards und zukünftige Entwicklungen in den medizinischen Spezialgebieten. 4. Ausgabe. 1995

${ }^{40}$ Mall RW, Medeiros M. Objective evaluation of results of a pulmonary rehabilitation program in a community hospital. Chest 1988; 94: $1156-1160$

${ }^{41}$ Agle DP, Baum GL, Chester EH et al. Multidiscipline treatment of chronic pulmonary insufficiency. Psychosom Med 1973; 35: 41 - 49

${ }^{42}$ Lacasse Y, Wong E, Guyatt GH et al. Meta-analysis of respiratory rehabilitation in COPD. Lancet 1996; 348: 1115-1119

${ }^{43}$ Wempe JB, Wijkstra PJ. The influence of rehabilitation on behaviour modification in COPD. Patient Education and Counseling 2004; 52: 237-241

${ }^{44}$ Casaburi R. Exercise training in chronic obstructive lung disease. Principles and Practices of Pulmonary Rehabilitation. Philadelphia: Saunders 1993; 23: $317-321$

${ }^{45}$ Berry MJ, Rejeski WJ, Adair NE et al. Exercise rehabilitation and chronic obstructive pulmonary disease stage. Am J Respir Crit Care Med 1999; 160: 1248 - 1253

${ }^{46}$ Satta A. Exercise training in asthma. J Sports Med Phys Fitness 2000; 40: $277-283$

${ }^{47}$ Büchi S, Brändli O, Klingler K et al. Stationäre Rehabilitation bei Patienten mit chronisch obstruktiver Lungenkrankheit (COLK): Effekte auf körperliche Leistungsfähigkeit, psychisches Wohlbefinden und Lebensqualität. Schweiz Med Wochenschr 2000; 130: 135-142

48 Petro W. Zur Praxis der Patientenschulung bei obstruktiven Atemwegserkrankungen (Asthma und COPD) in Deutschland. Pneumologie 2002; 56: 165 - 166

49 Behnke M, Taube C, Kirsten D et al. Die Langzeitwirkungen eines häuslichen Gehtrainings bei Patienten mit schwergradiger COPD. Pneumologie 2000; 54 (Sonderheft 1): S56

50 Salman GF, Mosier MC, Beasley BW et al. Rehabilitation for patients with chronic obstructive pulmonary disease: meta-analysis of randomized controlled trials. J Gen Intern Med 2003; 18: 213-221

${ }^{51}$ Lecheler J, Biberger A, Pfannebecker B. Asthma und Sport. Theoretische Grundlagen und praktische Handlungsanweisungen. Berchtesgaden: INA-Verlag, 1997

${ }^{52}$ Clark CJ. The role of physical training in asthma. Principles and Practice of pulmonary rehabilitation. Casabury and Petty. Philadelphia: Saunders, 1993

${ }^{53}$ Kirsten D, Taube C, Lehnigk B et al. Exercise training improves recovery in patients with COPD after an acute exacerbation. Respir Med 1998; 92: 1191 - 1198

${ }^{54}$ Behnke M, Schwertfeger I, Froreich K von et al. Monitoring home-based exercise training in patients with stable COPD. Am J Respir Crit Care Med 2002; 165 (suppl): A16

${ }^{55}$ Behnke M, Kirsten D, Jörres RA et al. Home-based exercise training in patients with COPD - Global effects. Am J Respir Crit Care Med 2000; 161 (suppl.): A254

56 Oga T, Nishimura K, Tsukino M et al. Relationship between different indices of exercise capacity and clinical measures in patients with chronic obstructive pulmonary disease. Heart Lung 2002; 31: $374-381$

${ }^{57}$ Behnke M, Taube C, Kirsten D et al. Home-based exercise is capable of preserving hospital-based improvements in severe chronic obstructive pulmonary disease. Respir Med 2000; 94: 1184-1191

${ }^{58}$ Behnke M, Jörres RA, Kirsten D et al. Clinical benefits of a combined hospital and home-based exercise programme over 18 months in patients with severe COPD. Monaldi Arch Chest Dis 2003; 59: 44-51

${ }^{59}$ Garcia-Aymerich J, Farrero E, Felez MA et al. Estudi del Factors de Risc d'Aguditzacio de la MPOC investigators. Risk factors of readmission to hospital for a COPD exacerbation: a prospective study. Thorax 2003; 58: 100- 105

${ }^{60}$ Ries AL, Kaplan RM, Myers R et al. Maintenance after pulmonary rehabilitation in chronic lung disease: a randomized trial. Am J Respir Crit Care Med 2003; 167: 880-888

61 Puente-Maestu L, Sanz ML, Sanz P et al. Comparison of effects of supervised versus self-monitored training programmes in patients with chronic obstructive pulmonary disease. Eur Respir J 2000; 15: $517-525$

${ }^{62}$ Worth H, Buhl R, Cegla U et al. Leitlinie der Deutschen Atemwegsliga und der Deutschen Gesellschaft für Pneumologie zur Diagnostik und Therapie von Patienten mit chronisch obstruktiver Bronchitis und Lungenemphysem (COPD). Pneumologie 2002; 56: 704-738
${ }^{63}$ Worth $\mathrm{H}$, Meyer A, Folgering $\mathrm{H}$ et al. Empfehlungen der Deutschen Atemwegsliga zum Sport und körperlichen Training bei Patienten mit obstruktiven Atemwegserkrankungen. Pneumologie 2000; 54: $61-67$

${ }^{64}$ Hunter DJ, Fairfield G. Disease management. BMJ 1997; 315: 50 - 53

${ }^{65}$ Bodenheimr T. Disease management - promises and pitfalls. N Engl J Med 1999; 340: $1202-1205$

${ }^{66}$ DeBusk RF, Miller NH, Superko HR et al. A case-management system for coronary risk factor modification after acute mycardial infarction. Annals of Internal Medicine 1994; 120: 721 - 729

${ }^{67}$ Kühn H. Disease Management - Erfahrungen aus den USA. Zeitschrift für Allgemeinmedizin 2002; 78: 175-180

68 Pfeifer M, Werner B, Magnussen H. Telemedizinische Betreuung von Patienten mit chronischen Atemwegserkrankungen. Med Klin 2004; 99: $106-110$

${ }^{69}$ Finkelstein J, Cabrera MR, Hripcsak G. Internet-based home asthma telemonitoring: can patients handle the technology. Chest 2000; 117: $148-155$

${ }^{70}$ Dale J, Connor S, Tolley K. An evaluation of the west Surrey telemedicine monitoring project. J Telemed Telecare 2003; 9 Suppl 1: 39-41

${ }^{71}$ Celler BG, Lovell NH, Basilakis J. Using information technology to improve the management of chronic disease. Med J Aust. 2003; 179: $242-246$

72 Borg GAV. Borg's perceived exertion and pain scales. 1st ed. Champaign, IL: Human Kinetics, 1998

73 Behnke M, Wewel AR, Kirsten D et al. Exercise training raises daily activity stronger than predicted from exercise capacity in patients with COPD. Respir Med im Druck,

${ }^{74}$ Scalvini S, Zanelli E, Domenighini D et al. Telecardiology community: a new approach to take care of cardiac patients. "Boario Home-Care" Investigators. Cardiologia 1999; 44: $921-924$

75 Molinari G, Reboa G, Frascio M et al. The role of telecardiology in supporting the decision-making process of general practitioners during the management of patients with suspected cardiac events. JTelemed Telecare 2002; 8: $97-101$

${ }^{76}$ Löllgen H, Steinberg T, Ertner C et al. Möglichkeiten der Telemedizin: Telefon-EKG in der Notfallmedizin. Intensivmedizin 1998; 35 : $630-640$

${ }^{77}$ Mair FS, Wilkinson M, Bonnar SA et al. The role of telecare in the management of exacerbations of chronic obstructive pulmonary disease in the home. J Telemed Telecare 1999; 5 Suppl 1: 66-67

${ }^{78}$ Ferrari M, Vangelista A, Vedovi E et al. Minimally supervised home rehabilitation improves exercise capacity and health status in patients with COPD. Am J Phys Med Rehabil 2004; 83: 337-343

${ }^{79}$ Mittag O, China C, Raspe H. Telefonische Nachsorge in der (kardiologischen) Rehabilitation: Ein Ansatz zum Disease Management durch Pflegepersonal. Praxis Klinische Verhaltensmedizin und Rehabilitation 2003; 61: 3-5

${ }^{80}$ Bundesministerium für Gesundheit. Strukturierte Behandlungsprogramme zur Verbesserung der Versorgung chronisch Kranker. 2002

${ }^{81}$ Scharnhorst J. Bedeutung und mögliche Zukunft telefonischer Nachsorgeprogramme aus Sicht der Krankenversicherungen - Organisation von Call-Centern. Praxis Klinische Verhaltensmedizin und Rehabilitation 2003; 61: $12-20$

82 Steele BG, Holt L, Belza B et al. Quantitating physical activity in COPD using a triaxial accelerometer. Chest 2000; 117: 1359-1367

${ }^{83}$ Steele BG, Belza B, Hunziker J et al. Monitoring daily activity during pulmonary rehabilitation using a triaxial accelerometer. J Cardiopulm Rehabil 2003; 23: 139-142

${ }^{84}$ Behnke M, Merkt B, Kirsten D et al. Does exercise training influence daily activity in patients with COPD? Am J Respir Crit Care Med 2000; 161: A753

${ }^{85}$ Bourbeau J, Julien M, Maltais F et al. Chronic Obstructive Pulmonary Disease axis of the Respiratory Network Fonds de la Recherche en Sante du Quebec. Reduction of hospital utilization in patients with chronic obstructive pulmonary disease: a disease-specific self-management intervention. Arch Intern Med 2003; 163: 585-591

${ }^{86}$ Ketelaars CA, Schlosser MA, Mostert R et al. Determinants of healthrelated quality of life in patients with chronic obstructive pulmonary disease. Thorax 1996; 51: 39-43

${ }^{87}$ Singh SJ, Smith DL, Hyland ME et al. A short outpatient pulmonary rehabilitation programme: immediate and longer-term effects on exercise performance and quality of life. Respir Med 1998; 92: 1146 - 1154

${ }^{88}$ Miyahara N, Eda R, Takeyama $\mathrm{H}$ et al. Effects of short-term pulmonary rehabilitation on exercise capacity and quality of life in patients with 
chronic obstructive pulmonary disease. Acta Med Okayama 2000; 54: $179-184$

${ }^{89}$ Nicholson C, Bowler S, Jackson C et al. Cost comparison of hospitaland home-based treatment models for acute chronic obstructive pulmonary disease. Aust Health Rev. 2001; 24: 181 - 187

${ }^{90}$ Burns M, Chang RSY, Lum E et al. Hospitalization rate of patients before and after a program of pulmonary rehabilitation. Am J Respir Crit Care Med 1996; 153: A127

91 Verband der privaten Krankenversicherung. Die private Krankenversicherung - Zahlenbericht 2000/2001. 2002

92 Biggar DG, Malen JF, Trulock EP et al. Pulmonary rehabilitation before and after lung transplantation. In: Casaburi R, Petty TL (eds). Principles and practice of pulmonary rehabilitation. Philadelphia: WB Saunders, 1993: 459-467

${ }^{93}$ Crouch R, MacIntyre NR. Pulmonary rehabilitation of the patient with nonobstructive lung disease. Respir Care Clin N Am 1998; 4: 59-70

${ }^{94}$ Walters EH, du Bois R. Immunology and management of interstitial lung diseases. London: Chapman \& Hall, 1995

${ }^{95}$ Konietzko N, Fabel H. Weißbuch Lunge 2000. Stuttgart: Georg Thieme Verlag, 2000: 39-40
${ }^{96}$ Keogh BA, Lakatos E, Price D et al. Pulmonary function testing in IPD. What does it tell us? Chest 1980; 78: 856-865

${ }^{97}$ Agusti AGN, Roca J, Rodriguez-Roisin R et al. Different patterns of gas exchange response to exercise in asbestosis and ideopathic pulmonary fibrosis. Eur Respir J 1988; 1: 510-516

${ }^{98}$ Harris-Eze AO, Sridhar G, Clemens RE et al. Oxygen improves maximal exercise performance in interstitial lung disease. Am J Respir Crit Care Med 1994; 150: 1616-1622

${ }^{99}$ Hansen JE, Wasserman K. Pathophysiology of activity limitation in patients with interstitial lung disease. Chest 1996; 109: 1566-1576

100 Leach RM, Davidson AC, Chinn S et al. Portable liquid oxygen and exercise ability in severe respiratory disability. Thorax 1992; 47: $781-789$

101 American Thoracic Society. Idiopathic pulmonary fibrosis: Diagnosis and treatment. Am J Respir Crit Care Med 2000; 161: 646-664

102 Behnke M, Schwertfeger I, Zimmermann I et al. Home based exercise training in patients with interstitial lung disease. Eur Respir J 2003; 22 (suppl.): $164 \mathrm{~s}$ 